\title{
Accreditation Policy of Higher Education: A Case Study of Japan
}

\author{
Farida Ibrahim Ramadan \\ Ministry of Higher \\ Education Egypt, \\ Kafrelsheikh University, \\ Egypt
}

\author{
Katsuhiro Umemoto \\ Graduate School of \\ Knowledge Science, \\ JAIST Japan
}

\author{
Meng Lanfang \\ JAIST, Japan, \\ Zuraidah Zaab \\ UiTM Malaysia, Sabah \\ Campus,
}

\author{
Amira Ramadan Abd El- \\ Hadi \\ Kafrelsheikh University, \\ Egypt \\ Om Elsaad Abu Elaneen \\ Hatata, \\ Kafrelsheikh University, \\ Egypt
}

\begin{abstract}
The quality of a country's higher education $(H E)$ and its assessment, monitoring and accreditation policies are a key not only to its social and economic wellbeing but are also a determining factor in the status of that HE system at the international level. In Japan, quality of education has been an important issue ever since the modern education system was founded in the late $19^{\text {th }}$ century; as Japan worked to catch up rapidly with western industrial countries. Since, the 1950s, the Japanese HE has been accustomed to assurance for the establishment of new universities and colleges. Thus quality assurance (QA) policy as such is not a new responsibility for Japanese higher education institutions (HEIs). However, the concepts of QA and accreditation are still new. Since 2004, the quality assurance and accreditation system (QAAS) requires that all universities in Japan are to undergo an accreditation process, once every seven years by certified agencies. Accordingly, this paper addresses a brief summary of QA framework of Japanese HE, which we divide simply into main distinct features; the self-evaluation, Standards for Establishing Universities (SEU) and the certified evaluation organizations. As of 2008, there are three institutional accreditation bodies in Japan; Japan University Accreditation Association (JUAA); National Institutional for Academic Degrees and University Evaluation (NIAD-UE); and Japan Institution for Higher Education Evaluation (JIHEE). This paper aims at presenting how these three certified agencies build up their accreditation knowledge base. Moreover, this paper proposes a theoretical model (ERSS Model) of accreditation policy making process in Japan. This model consists
\end{abstract}

of four phases; Evaluation, Recognition, Synthesization and Socialization.

\section{Introduction}

The concept of QA refers to "the intension and activities planned to assure quality" [1]. Parri [2] concluded that quality in $\mathrm{HE}$ may refer to exceptionality and excellence, zero errors, fitness for purpose, transformation and reshaping, threshold or enhancement. Therefore, QA in HE should be comprehensive and examine inputs, processes, and outputs [3].

By the end of $19^{\text {th }}$ century the transition from elite to mass HE [4] created many fundamental changes as well as challenges facing decision makers in the field of HE that the real 'pre-quality era' started [5]. Therefore, in the 1990s there was a significant change in the quality mechanisms and this decade can be seen as the decade of quality in HE. From this time on HEIs are required to demonstrate, through their institutional leaders and to express in comparable measures, the quality of its activities because governments stressed in value for-money and fitness- for-purpose [6].

Consequently, there has been an impressive rise in the number of national, regional or specialized QA and accreditation agencies in the past two decades. Countries have increasingly established national regulatory frameworks that evaluate and monitor quality in HE. Each country acquires policy/knowledge of QA and accreditation of $\mathrm{HE}$ through four kinds or channels; global, regional, international, and national transfer [7].

In Japan, the Ministry of Education, Culture, Sports, Science and Technology (MEXT) has been encouraging the creation of professional accrediting organizations [8]. Accordingly, since April 1, 2004, 
in order to improve the quality of and raise the standards for education and research, all universities and colleges, junior colleges, and other institutions of HE have been required to undergo comprehensive evaluations every 7 years conducted by the evaluation bodies (certified evaluation agencies) accredited by the Minister of Education with regard to the state of education and research, organizational management, facilities and equipment of evaluated institutions [9].

\section{Backgrounds}

\subsection{Japanese HE system}

The HE system in Japan consists of universities, junior colleges and colleges of technology. In addition, specialized training colleges offering postsecondary courses are regarded as one type of HE institution [10]. Japan has three kinds of universities; national, public and private universities.

Around three quarters of HEIs are privately owned and operated. However, the public universities have two thirds of graduate students who largely major in science and engineering [11]. Since 2004, an independent corporation status was given to all national universities (NUCs). As a result, all national universities have been organized as corporation aims to improve their independence and autonomy in order to revitalize education and research activities.

\subsection{QA framework in Japan}

Since 1956, QA of Japanese HE was based mainly on ministerial control over the establishment of new HEIs. This system was called an "ex-ante evaluation" based on the "Standards for Establishment of Universities" (SEU) set by the government. The SEU as a ministerial ordinance stipulates that it shall contain the minimum standards in order to establish a university. The structure of SEU is divided into four regulations;

- Regulations concerning the basic framework such as qualifications for admission, duration of study, and organizations;

- Regulations stipulating the minimum standards for human and material resources such as faculty, facilities and equipment;

- Regulations stipulating the norm for educational activities in university;

- Regulations for taking courses and requirements for graduation.

By the beginning of the 1990s, Japanese universities have been in the process of significant reform, often called the third wave in the history of
Japanese HE. This reform required to conduct selfmonitoring and self-evaluation. In 2002, a new system called "Certified Evaluation System" was launched, requiring all national, public and private universities, junior colleges and colleges of technology to undergo periodic evaluation, every seven years, by an evaluation agency certified by MEXT [12].

For Japanese HEIs to be more autonomous and independent, they must have an Internal Quality Assurance System (IQAS) directed towards reform and improvement through self-study (figure 1 shows the relationship between self-study and certified evaluation process). They must engage in active QA based on self-study results rather than simply yield to accreditation from the national government or a third-party.

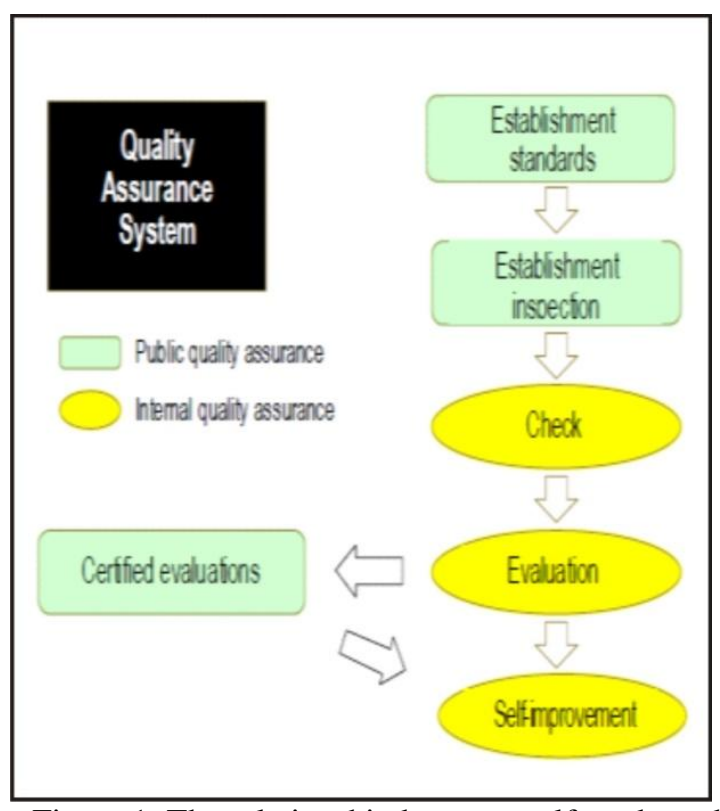

Figure 1. The relationship between self-study and certified evaluation system [13]

The HEI itself is most responsible for IQAS that ensures its future and secures social trust. Self-study verifies whether performance meets targeted goals; what the problems are if goals have not been achieved; finding the root of the problem and taking positive action for solutions. It is essential to clarify goals, make diligent plans and efforts to realize these goals, and steadily improve quality based on appropriate feedback from checks/evaluations and reform. For the IQAS to function effectively, the Plan-Do-Check-Act (PDCA) cycle should continue to rotate effectively at universities (figure 2). Each time this cycle is implemented, it leads to improvement and reform, forming an upward spiral. Each of our cases built its own standard scheme to evaluate whether the IQAS of each HEI is effectively functioning. 


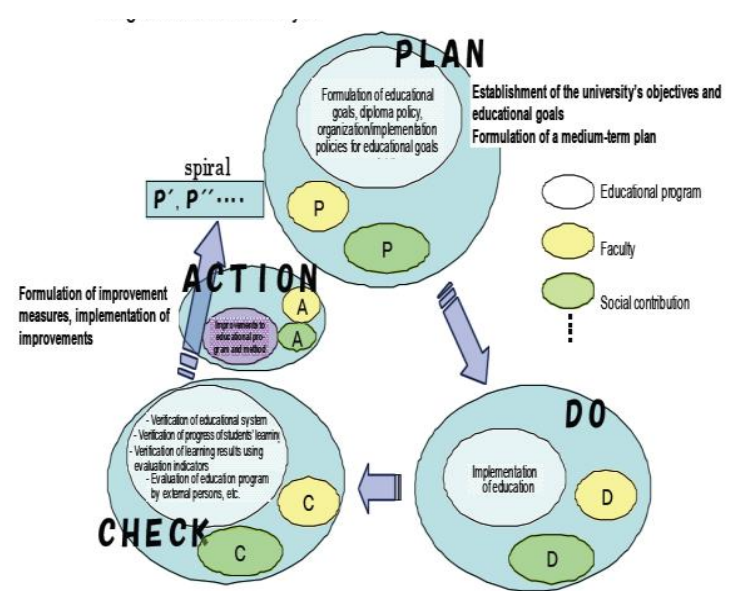

Figure 2. The PDCA cycle [13]

\subsection{Certified evaluation and accreditation agencies}

As of 2008, there are three institutional accreditation bodies in Japan; Japan University Accreditation Association (JUAA); National Institutional for Academic Degrees and University Evaluation; and Japan Institution for Higher Education Evaluation (JIHEE). In our discussion section of this paper, we develop a theoretical model of accreditation policy making process in Japan. The next section elucidates a background of these three distinctive entities for accreditation in Japan

- The Japan University Accreditation Association (JUAA);

JUAA is an independent organization established in 1947 under the sponsorship of 46 national, local public and private universities as Japan's first HE accreditation organization that based on the model of United States accreditation agencies [14]. Officially, in 2004, JUAA received the status as a certified evaluation agency for universities, and in 2006 for junior colleges and law schools.

JUAA is a membership-based organization, consisting of four-year national, local public and private university members. Its membership is divided into two categories; full membership, those are universities that have obtained their accreditation status through evaluation by the JUAA's standing Accreditation Committee, and associate membership. They are the members who supported the establishment of JUAA, its project and activities. This second kind of membership can be obtained without evaluation.

- The National Institution for Academic Degrees and University Evaluation (NIAD-UE);

In 1991, the University Council made its recommendation that the system of self-review and self-evaluation of activities at each university and college should be introduced for the purpose of improving university education and research. As a result, MEXT started external or third-party review system by the establishing NIAD-UE.

NIAD-UE is an independent evaluation and accreditation organization certified by the Minister of MEXT as evaluation agency in Japan that plays a leading role in development of third-party evaluation of universities, junior colleges, colleges of technology and professional graduate schools. NIAD-UE established two optional evaluation kinds; "the conditions of research" and "education offered to those other than full-time student" and also conducts evaluations at the request of higher education institutions [15].

- Japan Institution for Higher Education Evaluation (JIHEE);

Since July 2005, the JIHEE was authorized by the MEXT as one of the certified evaluation and accreditation organizations. JIHEE evaluates the situation in which educational and research activities are conducted at private universities and assisted in their self-initiated endeavors to enhance and improve the quality of Japanese HE.

\section{Methodology}

The analyzed materials have various forms such as; governmental official documents, press releases, and interviews' collected data. Our strategy in this paper is an in-depth qualitative case study approach. By using a case study approach, we focus on both the QA and accreditation policy for Japanese HE system, also on the context in which this policy is exists and transferred. The authors used the Qualitative Data Analysis Software (MAXQDA) as a tool in analyzing the collected data.

The procedures for the data collection and analysis process are interwoven within an iterative cycle consisting of interview-analyze-refineinterview. The total number of interviewees is 13 . Three of them are academics at excellence centers in Japanese HEIs. Two are JUAA's experts. Four are from NIAD-UE. Two are MEXT's experts, and the rest are JIHEE's experts.

The interviews were semi-structured. The questions were asked about how Japanese Certified Organizations; JUAA, NIAD-UE and JIHEE make their own policy of accreditation.

Hence, our paper focuses on two main questions:

1- How do certified evaluation and accreditation agencies formulate their accreditation knowledge base?

2- What are the main stages of accreditation policy making of certified evaluation agencies in Japan? 


\section{Case analysis and discussion}

Based on our collected data and discussion with interviewees, we propose a theoretical model (ERSS Model) of the accreditation policy making process of the three agencies; JUAA, NIAD-UE and JIHEE. This model is similar to SECI model in shape, but distinguished itself into four distinctive phases (figure 3). The distinctive phases are; Evaluation, Recognition, Synthesization and Socialization. These four phases are occurring in a cyclical in two different acquisitions, sharing and transferring QA and accreditation knowledge trends; from regional and global knowledge trend (R/GK) to national knowledge (NK) and the opposite trend. These two trends also are in a spiral movement based on which distinctive phase it exist in.

The next part of this paper analysis these phases in details according to our supposed ERSS model and its two mutual trends.

\subsection{Evaluation phase}

In the evaluation phase, each agency depends on many different sources of QA and accreditation knowledge to review and evaluate its current policy according to the regional and global knowledge and trends in QA and accreditation policy. These sources form the agency knowledge base, such as;

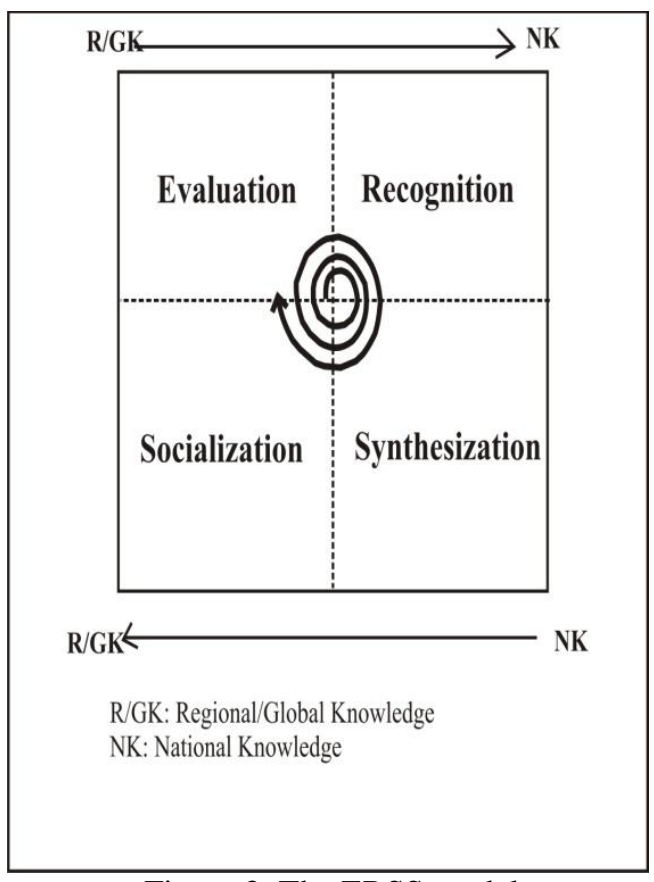

Figure 3. The ERSS model

Cultural knowledge; the cultural knowledge consists of basic knowledge around HE customers in Japan, realities about HE institutions, MEXT's policy for the future of HE and feedbacks knowledge from former cycles of accreditation process. In an interview with Professor Suzuki, a JUAA's expert, confirmed that;

JUAA formulates accreditation policy depended on MEXT's future view for Japanese $H E$.

The cultural knowledge contains not only Japanese HE knowledge, but also knowledge related to foreign systems of HE. In each accrediting agency there is a Research Department, the main role of the academics of this department is conducting advanced research about HE and QAS of other countries.

Expert's knowledge; the three agencies acquire new and different knowledge through joining workshops, seminars, meetings or conferences in foreign countries and inviting foreign experts to their agencies. For example, in June, 2010, NIAD-UE organized an open seminar titled "Aiming to Promote High Quality International Collaboration: the Japan-Europe QA Seminar". Members from nvao and Netherlands Organization for International Cooperation in Higher Education (Nuffic) participated in this seminar under the support of MEXT.

Experts tacit knowledge; benchmarking knowledge of experts who conduct sites visits is considered a main resource for building each agency's knowledge base.

Network knowledge; the three agencies acquire different foreign QA and accreditation knowledge through their membership or joining globalised networks such as:

- International Network for Quality Assurance Agencies in Higher Education (INQAAHE);

- $\quad$ Asia-Pacific Quality Network (APQN);

- Quality Assurance Agency for Higher Education (QAA).

In an interview with one of the NIAD-UE experts, he stated that;

I am one of NIAD-UE experts who always join INQAAHE. Through its annually conferences, symposiums, workshops and even during having meals, my discussion with experts worldwide gives me very deep understanding about many topics related to how would national system of accreditation works in the context of global HE world.

In another interview with one of the JIHEE experts he confirmed that;

We here are experts of JIHEE, a just new accrediting agency, seek developing it as fast as we can. One of our recent aims is to join these regional and global networks specializing in $Q A$ and accreditation culture of HE that will enable us to do so.

Communities of Practice (CoP) knowledge;

- A mutual knowledge is acquired through making a memorandum of understanding between most of Japanese accrediting agencies and other foreign agencies. For example, the memorandum of 
understanding which was made between Accreditation Organization of the Netherlands and Flanders (nvao) and NIAD-UE.

- Cooperation among Japan, China and Korea QA organizations, in March 2010.

- Cooperation with Hong Kong Council for Accreditation of Academic and Vocational Qualifications (HKCAAVQ).

- The three agencies demonstrated that they have a kind of mutual meeting with each other and with MEXT regarding accreditation policy.

IQAS knowledge; each agency seeks acquiring knowledge covering the QAS in each HEI which named IQAS. This system simply shows how each institution build up its goals and plans, do these plans, chick their realization that finally realize accreditation standards.

In general, for each case, the self-study reports, which each HEI conducts in the beginning of its accreditation process, provide a foundation for peer or external-review (evaluation team). In addition to self-assessment feedback knowledge is really important not only for the certified evaluation organization but also for the HEI itself.

Agency's package, website and surveys; accrediting agencies depend on their knowledge package and website in sharing their knowledge with other agencies and their members. Moreover, agencies conduct surveys which aim at having a whole vision about HEIs and what difficulties facing these institutions to have the accreditation certificates.

In an interview with one of the NIAD-UE experts he stated about surveys,

After its first-cycle of certified accreditation, NIADUE conducted multi-choice, five-levels, written questionnaires at HEIs (universities and junior colleges) subjected to institutional certified evaluation and accreditation from 2005-2008.

Also, in an interview with one of JIHEE experts, he stated that;

In the beginning of establishing our JIHEE organization, actually the Private Universities Corporations (PUCs) request this establishment because the unique nature of private universities (PUs) in Japan. In our beginning we conducted surveys for all intended members. These surveys and interviews aimed at having a whole vision about what these corporations and their institutions seek for in the QA way. Moreover, these surveys made us able to have our first edition of our standards.

Round table discussion with members; in a case of a membership structure organization such as JUAA and JIHEE, members have a role in providing the accrediting agency by their opinion.

In an interview with one of the JUAA's experts he confirmed that;
Of sure, the members pay fees for this membership. That is clearly means that they should have a role in making our working policy/ standards. But what really happens that, after we conduct both of the evaluation and recognition process for our standard/s, we invite the members to a discussion, a round table discussion. As a result, they agree for it because they feel that they did a role in making such a policy. We here in JUAA consider our membership structure is a unique structure, because we started as a first Japanese independent agency by this membership structure.

Regional/Global projects knowledge such as OECD's Assessment of Higher Education Learning Outcomes (AHELO);

The majorities of the interviewees confirmed that the recently policy of MEXT seeks develop internationalization of Japanese HES through engaging in both regional and global projects. As a part of this engagement, QA and accreditation policy is transferred among the participating countries. Our three cases believe that the participating of these kind of global projects save both money and effort for them to have great new knowledge. In an interview with one of the NIAD-UE experts she stated that;

The recent policy of MEXT is to enforce its internationalization policy through the Asia-Pacific region. It is easy to notice that even in its homepage site.

The previous present of NIAD-UE, Shinichi Hirano, was one of the Japan-China-Korea committee members for Promoting and Exchange and Cooperation among universities, this meeting had been held several times since 2009. This meeting aimed at quality-assured exchanges.

Moreover, NIAD-UE as one of our cases will join AHELO project. The OECD is carrying out a feasibility study to see if it is practically and scientifically feasible to assess what student in $\mathrm{He}$ know and can do upon graduation. So, AHELO assessment aims to be direct evaluation of student performance at the global level and valid across diverse cultures, languages and different types of institutions. In an interview with one of NIAD-UE's experts he states that;

The recent interesting topic in accreditation criteria is the student learning outcomes. I think we will gain more and more and understand how the world thinks about it, and how are different countries think about its realization through our joining to this great project.

HEIs evaluation knowledge; since adapting the NUCs for national universities, the National University Corporation Evaluation Committee (NUCEC), under the MEXT, is responsible for performance-based evaluation of 86 NUCs and 4 inter-university research institute corporations in respect of their attainment of their 6-year mid-term 
plans and annual plans for education, research and management. At the same time, the NIAD-UE is appointed to undertake evaluation on the attainment of mid-term objectives and the present conditions in terms of education and research [16]. These kinds of knowledge related to the evaluation of HEIs internal conditions are considered main inputs and vital source for each accrediting agency knowledge base.

Agency's recognition feedback; each accrediting agency strives to develop its own distinctive evaluation and accreditation system through the recognition process. The result of this process is considered another feedback for accreditation knowledge base. Interviewees were eager to discuss student learning outcomes and how their agencies try to adopt more new categories of standards related to this branch. Also, how the accreditation process could embrace measures of student learning as indicators of Japanese quality of HE. The next phase shows how this process happens in our cases.

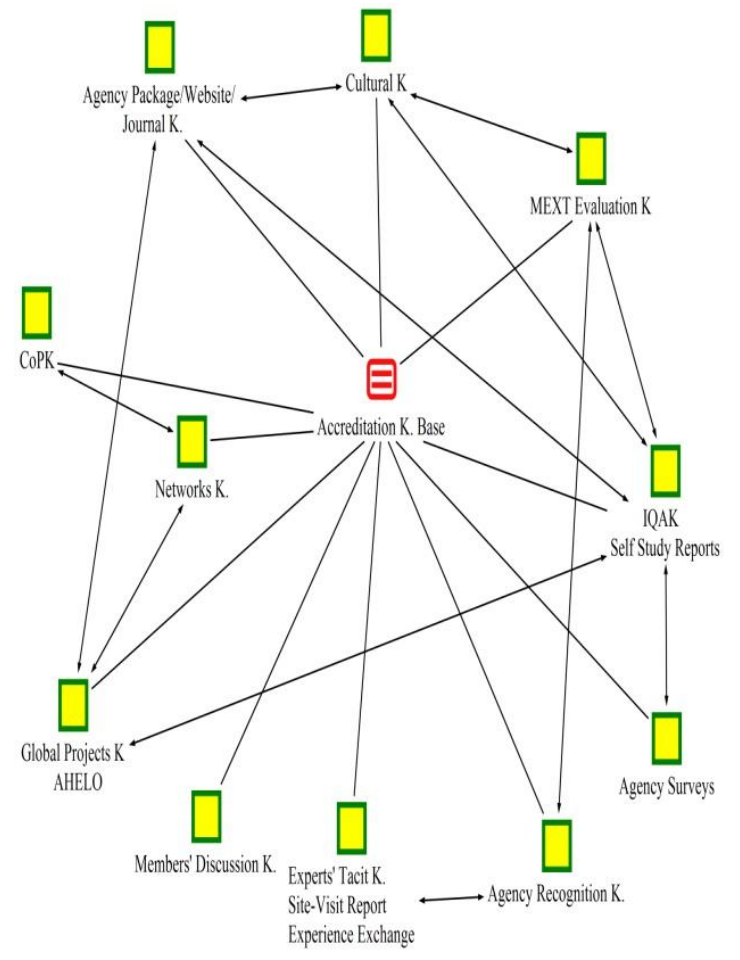

CoPK: Communities of Practice knowledge

IQAK: Internal Quality Assurance Knowledge

AHELO: Assessment of Higher Education Learning

Outcomes project

Figure 4. Accreditation knowledge base in Japan

According to these sources of QA and accreditation knowledge that shown above and are introduced in figure 4 (by using the visual tools of MAXQDA program) as knowledge base of each of our cases. JUAA, NIAD-UE, and JIHEE use this QA and accreditation knowledge base in conducting review for its recent policy and evaluate it.
According to this review and evaluation phase, each agency decides which side of defect exists in its recent evaluated policy. Then, the recognition phase supposes what solutions are suitable for this defect, as it will be shown in the next part.

\subsection{Recognition phase}

In the American culture of the recognition process, both the federal government, through the United States Department of Education, and the Council for Higher Education Accreditation (CHEA) recognize accrediting organizations. Federal, as distinct from CHEA, recognition aims to assure that the standards of accrediting organizations meet expectations for institutional and program participation in federal initiatives, such as student aid. The recognition process in the federal government and CHEA is conducted for considering the eligibility status of new and continuing accrediting organizations.

In this paper, we mean by recognition phase the change of recognized scope of accreditation policy of continuing accrediting organization. Based on our interview results we recognized that each accrediting agency, after conducting its own review and evaluation, appoints a recognition committee.

Each agency supply its recognition committee by its own accreditation knowledge base and its evaluation phase result. Moreover, each committee members acquire more knowledge through their round-table brainstorming discussion with others from different agencies and with members of National Institute for Educational Policy Research (NIER). NIER provides MEXT with needed data to plan and design educational policy.

As a result, the recognition committee presents a report of the new modification of standard/s to the agency's Board of Directors.

\subsection{Synthesization phase}

In this phase, after recognition committee of each agency finished its work and submits its result to the Board of Directors of each agency. Each agency reformulates its new policy as standers for accreditation, which will be followed by any HEI wishes to be accredited. Therefore, we can conclude that after evaluation and recognition processes the Synthesization process is happens under the nationalization trends. Or we can say that the proposed new policy should serve Japanese domestic HE system and simultaneously push it towards a competitive globalised case. This is simply happens during the accreditation process itself. At the same time, the new formulated policy/standards is/are ready to be shared with other specialized QA experts, agencies and networks under the trend of transferring 
the national knowledge to regional/global knowledge.

\subsection{Socialization phase}

The Socialization phase is the last phase of our proposed theoretical model. We suggest that the new policy/standards is/are tested through the accreditation process itself, which accesses mainly in HEIs. The final feedback knowledge of this stage, accreditation process, is considered as new inputs for a new cycle of accreditation policy making process which begin with evaluation of the current policy according to the regional/global trend of QA and accreditation policy.

The socialization phase consists of two main parts. First, the accreditation process which happens in each HEI seeking accreditation. Second, the feedback of the accreditation process is considered a critical input for future recognition process.

For the three cases, JUAA, NIAD-UE, JIHEE, the accreditation process is conducting at the same way. Following is the distinctive steps of the accreditation process for the three cases;

- HEI conduct self-assessment and produce a report according to the report guidelines of each agency.

- The agency process, mostly the method of the evaluation of the self-assessment reports is based on document analysis and site visit. Document analysis examines the submitted self-assessment report in according to each agency evaluation manual. The site visit is the stage to interview staff/student and inspect university's faculties, based on the guidelines for site visit, in order to scrutinize in greater depth and verify issues which could not be determined during the document analysis. So, the agency process is summarized in the self-assessment reports analysis, and the site visits for HEIs.

- The judgments; each agency judges whether the university as a whole meets each standard or not and gives its reasons for its judgment. When any one of the standards is not being met, the university is judged as unsatisfactory. These result for the three cases are made public.

- Universities may apply for the next evaluation and accreditation process after an interval of at least five academic years. In a case of universities that judged failing the standards. The agency conducts a supplementary review. Universities may apply for this supplementary review within two years of the evaluation year.

\section{Conclusion}

The main conclusion of our paper is the development of our theoretical model of Japanese accreditation policy making process. The ERSS Model consists of four main phases; Evaluation, Recognition, Synthesization and Socialization. These four phases work in a cyclical form begins with Evaluation, through Recognition and Synthesization and ends by Socialization. This cycle works within two QA and accreditation policy transfer trends; from regional/global knowledge to national knowledge and from national knowledge to regional/global knowledge.

\section{Acknowledgements}

We would like to thank the anonymous reviewers of this paper. The first author is highly grateful to the Egyptian Government (Ministry of Higher Education (sponsor) for providing financial support.

\section{References}

[1] M. Segers, and F. Dochy, "Quality Assurance in Higher Education: Theoretical Considerations and Empirical Evidence", Studies in Educational Evaluation, 22-2, Elsevier, 1996, p. 119.

[2] J. Parri, "Quality in Higher Education", VADYBA Management, 2-11, 2006, pp. 107-111.

[3] M. Frazer, "Quality Assurance in Higher Education", in A. Craft, (eds.) Quality Assurance in Higher Education, Proceedings of an International Conference, Routledge, London, 1992.

[4] M. Trow, "Problems in the Transition from Elite to Mass Higher Education". In OECD, Policies for Higher Education, the General Report on the Conference on Future Structure of Post-Secondary Education, OECD, Paris, 1974.

[5] P. Ewell, "The Quality Game: External Review and Institutional Reaction over Three Decades in the United States". In Westerheijden, D. et al. (Eds.), Quality Assurance in Higher Education, Trends in Regulation, Translation and Transformation, Springer, Dortrecht, 2007.

[6] A. Bernhard, Quality Assurance in an International Higher Education Area: A Case Study Approach and Comparative Analysis, VS Verlag, Wissenschaft, 2012.

[7] F. I. Ramadan, et.al, "Quality Assurance of Egyptian Higher Education: A Policy Transfer Perspective", Literacy Information and Computer Education Journal, 21, 2011, pp. 338-349.

[8] R. Mori, Accreditation Systems in Japan and United States: A Comparative Perspective on Governmental Involvement. In S. P. O'Brien (ed.), Accreditation: Assuring and Enhancing Quality, Jossey-Bass, San Francisco, 2009.

[9] MEXT, Article 109-2 of the School Education Law. 
[10] Ministry of Education, Culture, Sports, Science and Technology [MEXT], Higher Education in Japan; www.mext.go.jp/English/highered/index.htm (2 June 2011).

[11] K. Yamamoto, "Corporation of National Universities in Japan: Revolution for Governance or Rhetoric for Downsizing", Financial Accountability \& Management, Blackwell Publishing Ltd, Oxford, May 2004, pp. 153-181.

[12] National Institution for Educational Policy Research (NIER), Higher Education in Japan; http://www.nier.go.jp/English/EducationInJapan/201109H E.pdf (7 June 2011).

[13] Japan University Accreditation Association (JUAA) homepage; http://www.juaa.or.jp (20 January 2012).

[14] Japan University Accreditation Association (JUAA), Toward the improvement of Higher Education Quality; http://www.juaa.or.jp (23 May 2012).

[15] NIAD-UE, The work of NIAD-UE 2010; www.niad.ac.jp/english/about/index.html (30 January 2012).

[16] Saito, T. University Evaluation Systems in Japan; www.ias.unu.edu/resource_center/TakahiroSaito.pdf (14 March 2012). 